Internist 2011 · 52:1147-1148

DOI 10.1007/s00108-011-2849-9

Online publiziert: 22. September 2011

(c) Springer-Verlag 2011

\author{
M. Reincke ${ }^{1} \cdot K$. Werdan ${ }^{2}$ \\ ${ }^{1}$ Medizinische Klinik Innenstadt, Klinikum der LMU, München \\ ${ }^{2}$ Universitätsklinik und Poliklinik für Innere Medizin III, Halle (Saale)
}

\title{
Internistische Erkrankungen in der Schwangerschaft II
}

Bei dem vorliegenden Oktoberheft von Der Internist handelt es sich um eine Fortsetzung: Internistische Erkrankungen in der Schwangerschaft I behandelte 2008 die Themen Bluthochdruck, Rhythmusstörungen, kardiale Vitien und Antikoagulation. In Internistische Erkrankungen in der Schwangerschaft II werden nun die ergänzenden Themen Niereninsuffizienz, Diabetes, Immunsuppression, Schilddrüsenerkrankungen sowie Alkoholmissbrauch und Rauchen behandelt. Alle dargestellten Erkrankungen führen bei ihrem Auftreten in der Schwangerschaft automatisch zur Klassifizierung als Risikoschwangerschaft.

Laut statistischem Bundesamt wurden im Jahr 2009 etwa 665.00o Kinder in Deutschland geboren. In Folge umfangreicher Qualitätssicherungsmaßnahmen ist es dabei über die letzten Dekaden gelungen, die mütterliche Sterblichkeit deutlich zu reduzieren. Sie liegt in Deutschland augenblicklich bei 7/100.00o [1] Auch die Perinatalsterblichkeit hat sich günstig entwickelt: Sie liegt inzwischen nur noch bei etwa 5/100o Geburten [2] Deutschland nimmt damit im internationalen Vergleich eine Spitzenpositionen und in Europa eine Position in den oberen $20 \%$ ein. Bei der Reduktion der Perinatalsterblichkeit spielt die frühzeitige Erkennung von Risikoschwangerschaften eine große Rolle. Eine Risikoschwangerschaft ist entsprechend der Mutterschaftsrichtlinien des Bundesausschusses der Ärzte und Krankenkassen [3] definiert (• Tab. 1). Verlässliche Angaben zur Prävalenz von Risikoschwangerschaft liegen von der Bayerischen Qualitätssicherung (BAQ, [4]) vor, die diese kontinuierlich seit über 20 Jahren dokumentiert. Demzufolge lag eine Risikoschwangerschaft im Jahr 1989 bei $59 \%$, 1999 bei $64 \%$ und 2009 bei unerwartet hohen $71 \%$ aller Schwangerschaften vor. Ähnliche Zahlen werden aber auch aus anderen Bundesländern gemeldet. Damit sind heutzutage 2 von 3 Schwangerschaften als Risikoschwangerschaft einzuschätzen. Diese deutliche Zunahme innerhalb von 2 Jahrzehnten überrascht und bedarf einer Erläuterung.

\section{(7) Heutzutage sind 2 von 3 Schwangerschaften als Risiko- schwangerschaft einzuschätzen}

Zunächst wird der Zuwachs teilweise durch den fortlaufenden Anstieg des Alters der schwangeren Frauen erklärt, da $\mathrm{ab}$ einem mütterlichen Alter von 35 Jahren eine Schwangerschaft definitionsgemäß als Risikoschwangerschaft eingestuft wird. Ein weiterer Faktor liegt in der aktiven Erhebung von Risikokonstellationen, wie z. B. dem Screening nach Gestationsdiabetes. Vorsorgemaßnahmen wie die hochauflösende Ultraschalluntersuchung verbessern die antenatale Aufdeckung auch geringer Fehlbildungen, die automatisch zum Status einer Risikoschwangerschaft führen. So gesehen reflektieren diese Zahlen insbesondere auch die erfolgreichen Bemühungen um eine Intensivierung der Risikovorsorge.

Die in diesem Heft behandelten Erkrankungen erfordern eine konsequente
Begleitung und Therapieanpassung in der Schwangerschaft. Die Übersichten sollen den behandelnden Internisten in die Lage versetzen, das Richtige zu tun und das Falsche zu unterlassen. Deshalb wurde besonders auf eine praxisnahe Darstellung Wert gelegt.

Die Übersichtarbeit von Lechner, Lohr und Seissler stellt eine aktuelle Übersicht zum Thema Gestationsdiabetes dar. Nach aktuellen Untersuchungen ist jede 5 . Schwangere von einem Diabetes mellitus betroffen. Die Ergebnisse mehrerer prospektiver Studien haben zu einer Anpassung der Blutzuckergrenzwerte geführt. So ist bei einem Nüchternblutzucker $>92$ $\mathrm{mg} / \mathrm{dl}$ (bzw. 5,1 mmol/l), einem Blutzucker $>180 \mathrm{mg} / \mathrm{dl}(10,0 \mathrm{mmol} / \mathrm{l}) 1 \mathrm{~h}$ nach oraler Glukosebelastung und einem 2-hWert $>153 \mathrm{mg} / \mathrm{dl}(8,5 \mathrm{mmol} / \mathrm{l})$ im venösen Plasma von einem Gestationsdiabetes auszugehen.

Während das Thema Gestationsdiabetes in den letzten Jahren eine hohe Aufmerksamkeit erzielt hat, kann dies für das Thema Schilddrüsenfunktionsstörungen in der Schwangerschaft nicht in gleicher Weise behauptet werden. Dabei sind eine ausreichende Jodversorgung sowie die adäquate Dosisanpassung einer Thyroxintherapie bei hyopthyreoten Frauen von großer gesundheitlicher Relevanz für das werdende Kind. In dem Artikel von Dagmar Führer werden die neuesten Erkenntnisse und Empfehlungen zu diesem Thema umfangreich dargestellt.

Die Prävalenz einer vorbestehenden Niereninsuffizienz ist mit $0,2 \%$ aller Schwangerschaften relativ gering. Be- 
Tab. 1 Definition von Risikoschwangerschaften nach den Mutterschaftsrichtlinien des Bundesausschusses der Ärzte und Krankenkassen [3]

\begin{tabular}{|c|c|}
\hline \multicolumn{2}{|l|}{ Anamnestische Risiken } \\
\hline $\begin{array}{l}\text { Schwere Allgemeinerkrankungen } \\
\text { der Mutter }\end{array}$ & $\begin{array}{l}\text { Erkrankungen der Niere und der Leber oder schwere } \\
\text { Adipositas (Fettleibigkeit) }\end{array}$ \\
\hline Alter & Erstgebärende unter 18 Jahren oder über 35 Jahre \\
\hline \multirow{6}{*}{$\begin{array}{l}\text { Gynäkologische bzw. geburts- } \\
\text { hilfliche Faktoren }\end{array}$} & Totgeborenes oder geschädigtes Kind \\
\hline & $\begin{array}{l}\text { Vorausgegangene Entbindungen von Kindern über } \\
4000 \mathrm{~g} \text { Gewicht, hypotrophen Kindern (,small for date } \\
\text { babies"), Mehrlingen }\end{array}$ \\
\hline & Zustand nach Uterusoperationen \\
\hline & Komplikationen bei vorangegangenen Entbindungen \\
\hline & $\begin{array}{l}\text { Zustand nach Sterilitätsbehandlung, wiederholten } \\
\text { Aborten oder Frühgeburten }\end{array}$ \\
\hline & $\begin{array}{l}\text { Mehrgebärende über } 40 \text { Jahre, Vielgebärende mit } \\
\text { mehr als } 4 \text { Kindern }\end{array}$ \\
\hline \multirow{4}{*}{$\begin{array}{l}\text { Risiken in der aktuellen } \\
\text { Schwangerschaft }\end{array}$} & EPH-Gestose; Pyelonephritis \\
\hline & Anämie $<10 \mathrm{~g} / \mathrm{dl}$ \\
\hline & Diabetes mellitus bzw. Gestationsdiabetes \\
\hline & Blutgruppeninkompatibilität \\
\hline \multirow[t]{5}{*}{ Gynäkologische Faktoren } & Uterine Blutung \\
\hline & $\begin{array}{l}\text { Diskrepanz zwischen Uterus- bzw. Kindsgröße und } \\
\text { Schwangerschaftsdauer }\end{array}$ \\
\hline & $\begin{array}{l}\text { Drohende Frühgeburt (vorzeitige Wehen, Zervix- } \\
\text { insuffizienz) }\end{array}$ \\
\hline & Mehrlinge, pathologische Kindslagen \\
\hline & $\begin{array}{l}\text { Überschreitung des Geburtstermins bzw. Unklarheit } \\
\text { über den Termin }\end{array}$ \\
\hline
\end{tabular}

steht aber eine mäßige oder stark eingeschränkte Nierenfunktion, bedarf es aufgrund der hohen Inzidenz einer arteriellen Hypertonie mit konsekutivem Präeklampsierisiko einer sorgfältigen Beratung schon vor der Konzeption. Dies gilt insbesondere auch für die diabetische Nephropathie. Da die Gefahr einer Verschlechterung der Nierenfunktionseinschränkung gegeben ist, gilt der Pharmakotherapie der Hypertonie in der Schwangerschaft besonderer Augenmerk, wie im Beitrag von Siekierka-Harreis und Rump dargestellt.

Der Artikel von Walldorf, Dollmann und Seufferlein behandelt das Thema der immunsuppressiven Therapie in der Schwangerschaft. Das Spektrum verfügbarer Substanzen und die Indikationen von Immunsuppressiva haben sich in den vergangenen 10 Jahren kontinuierlich erweitert. Schwangerschaften unter Immunsuppressiva sind deshalb kei- ne Seltenheit mehr und bedürfen einer sorgfältigen präkonzeptionellen Planung und Therapiesurveillance während der Schwangerschaft.

Der letzte Beitrag von Thäle und Schlitt widmet sich den deletären Folgen von Alkohol- und Nikotinmissbrauch in der Schwangerschaft für die fetale Entwicklung. Die Zahl von 4000 Neugeborenen pro Jahr mit dem Vollbild eines fetalen Alkoholsyndroms spricht für sich. Es ist zu bedauern, dass trotz vielfältiger Anstrengungen der Bundeszentrale für gesundheitliche Aufklärung, der Berufsverbände und der wissenschaftlichen Gesellschaften eine Trendwende leider nicht in Sicht ist.

Liebe Leserinnen und Leser, wir als Herausgeber würden uns freuen, wenn Ihnen das vorliegende Heft die Mitbetreuung von Schwangeren mit Risikokonstellationen oder relevanten internis- tischen Begleiterkrankungen erleichtern und Ihnen praxisrelevante Informationen vermitteln würde, die in dieser kondensierten Form -so glauben wir - an anderer Stelle nicht zu finden sind.

Wir wünschen Ihnen beim Lesen dieser Ausgabe von Der Internist den erhofften Informationsgewinn.

Ihre

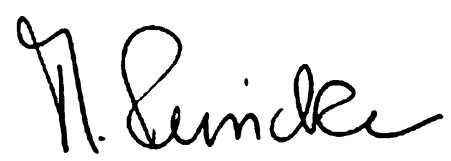

M. Reincke

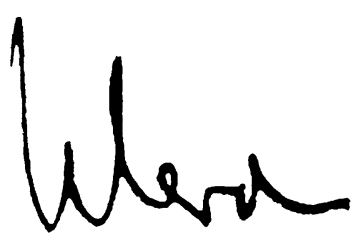

K. Werdan

\section{Korrespondenzadressen \\ Prof. Dr. M. Reincke}

Medizinische Klinik Innenstadt, Klinikum der LMU

Ziemssenstr. 1

80336 München

Martin.Reincke@med.uni-muenchen.de

\section{Prof. Dr. K. Werdan}

Universitätsklinik und Polikli-

nik für Innere Medizin III

Ernst-Grube-Str. 40

06120 Halle (Saale)

karl.werdan@medizin.uni-halle.de

\section{Literatur}

1. www.childinfo.org/files/Trends_in_Maternal_Mortality_1990_to_2008.pdf

2. http://ec.europa.eu/health/ph_information/dissemination/echi/echi_2_de.htm\#11

3. www.g-ba.de/downloads/62-492-70/RL_Mutter-2003-03-24.pdf

4. www.baq-bayern.de/downloads/files/2009_161_ gesamt_online.pdf 